\title{
Elucidating the relationship between arrhythmia and ischemic heterogeneity: an in silico study"
}

\author{
Sanjay R. Kharche* (IEEE and EMBS Member), Kairavi Desai, Christopher W. McIntyre.
}

\begin{abstract}
Dialysis causes blood flow defects in the heart that may augment electrophysiological heterogeneity in the form of increased number of ischemic zones in the human left ventricle. We computationally tested whether a larger number of ischemic zones aggravate arrhythmia using a 2D electrophysiological model of the human ventricle.

A human ventricle cardiomyocyte model capable of simulating ischemic action potentials was adapted in this study. The cell model was incorporated into a spatial 2D model consisting of known number of ischemic zones. Inter-cellular gap junction coupling within ischemic zones was reduced to simulate slow conduction. Arrhythmia severity was assessed by inducing a re-entry, and quantifying the ensuing breakup and tissue pacing rates.

Ischemia elevated the isolated cardiomyocyte's resting potential and reduced its action potential duration. In the absence of ischemic zones, the propensity in the 2D model to induce multiple re-entrant waves was low. The inclusion of ischemic zones provided the substrate for initiation of re-entrant waves leading to fibrillation. Dominant frequency, which measured the highest rate of pacing in the tissue, increased drastically with the inclusion of multiple ischemic zones. Reentrant wave tip maximum numbers increased from 1 tip (no ischemic zone) to 34 tips when a large number (20) of ischemic zones were included. Computational limiting factors of our platform were identified using software profiling.

Clinical significance. Dialysis may promote deleterious arrhythmias by increasing tissue level action potential dispersion.
\end{abstract}

\section{INTRODUCTION}

Ventricular arrhythmia post-dialysis is a routine complication [1]. A lack of the structure-function mechanism knowledge limits the development of novel effective therapies. It is thought that dialysis induced arrhythmias may be a manifestation of regionally heterogenous blood flow [2], giving rise to heterogeneous conduction. Heterogenous conduction has been shown to promote re-entrant wave breakup and fibrillation [3]. Whereas the genesis and persistence of arrhythmia due to an ischemic zone may be known [4], the relationship between multiple ischemic zones of clinically observed dimensions remains underexplored. We hypothesized that the heterogenous conduction is due to complex ischemic zones embedded in an otherwise electrophysiologically homogenous tissue (normal zone). Such as structure may lead to multiple re-entrant waves or fibrillation. This preliminary study presents a relationship between the numbers of ischemic zones and re-entrant waves in a $2 \mathrm{D}$ model of the human ventricle.

*This work was supported by Lawson Health Research Foundation (Canada). SRK is with Medical Biophysics, Western University and Lawson Health Research Institute, London Ontario, N6A 4G5, Canada. (phone: 519685-8500x56047; e-mail: Sanjay.Kharche@lhsc.on.ca). KD is a final year
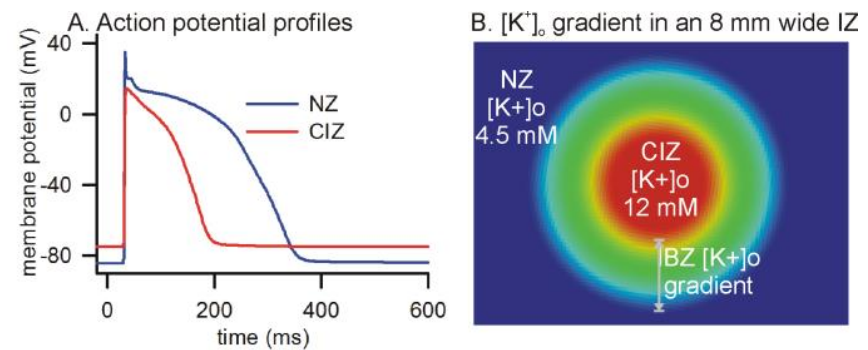

Figure 1. Simulated action potentials and ischemic zone morphology. A: Action potentials in the normal zone (NZ, blue) and central ischemic zone (CIZ, red). B: Ischemic zone morphology showing extracellular potassium gradient. The ischemic zone consists of a border zone that encompasses the gradient, and a central ischemic zone, CIZ.

\section{METHODS}

\section{A. Clinical data in cell modelling}

The 39 variable ionically detailed cell model of the human ventricle described by Carro et al. [5] was adopted to simulate cardiomyocyte action potentials under normal and ischemic conditions. The cell model electrophysiology was adapted by incorporating an additional adenosine triphosphate (ATP) sensitive potassium current, $\mathrm{I}_{\text {KATP }}$ as described previously [6]. To simulate the effect of ischemia, an increase of extracellular potassium, $\left[\mathrm{K}^{+}\right]_{\mathrm{o}}$, from $5.4 \mathrm{mM}$ to $12 \mathrm{mM}$, an increase of $\mathrm{I}_{\mathrm{KATP}}$ current conductance, $\mathrm{g}_{\mathrm{KATP}}$, from $0.04 \mathrm{pA} / \mathrm{pF}$ to $2.05 \mathrm{pA} / \mathrm{pF}$, and a $25 \%$ reduction of the upstroke (sodium and calcium) currents' conductances were implemented. The $\left[\mathrm{K}^{+}\right]_{\mathrm{o}}$ values were deduced from pre and post-dialysis serum measurements of 33 early dialysis patients. Realistic action potentials in the normal and ischemic zones are illustrated in Figure 1A.

\section{B. 2D human ventricle tissue model}

The 2D human ventricle model consisted of a structured finite difference grid spanning a large $80 \mathrm{~mm}$ x $80 \mathrm{~mm}$ sheet (400 x 400 nodes, $0.2 \mathrm{~mm}$ internode distance). Previously obtained CT imaging data [7] provided the dimensions and distribution of low perfusion ischemic zone regions. The data was incorporated into the model as circular $8 \mathrm{~mm}$ wide ischemic zones. Each ischemic zone consisted of a central ischemic zone surrounded by a $2 \mathrm{~mm}$ wide ischemic border zone. The central ischemic zone was assigned ischemic values as described above. Linear gradients of $\left[\mathrm{K}^{+}\right]_{\mathrm{o}}$, conductances, and ATP were implemented in the IBZ (Figure 1B). The diffusion in the normal zone was assigned a value of $D=0.004$ $\mathrm{mm}^{2} / \mathrm{ms}$ to produce a physiological conduction velocity of

undergraduate student in Medical Biophysics, Western University. CWM is a practicing physician (nephrologist) in London Health Sciences Centre, Lawson Health Research Institute, London Ontario, N6A 4G5, Canada. 

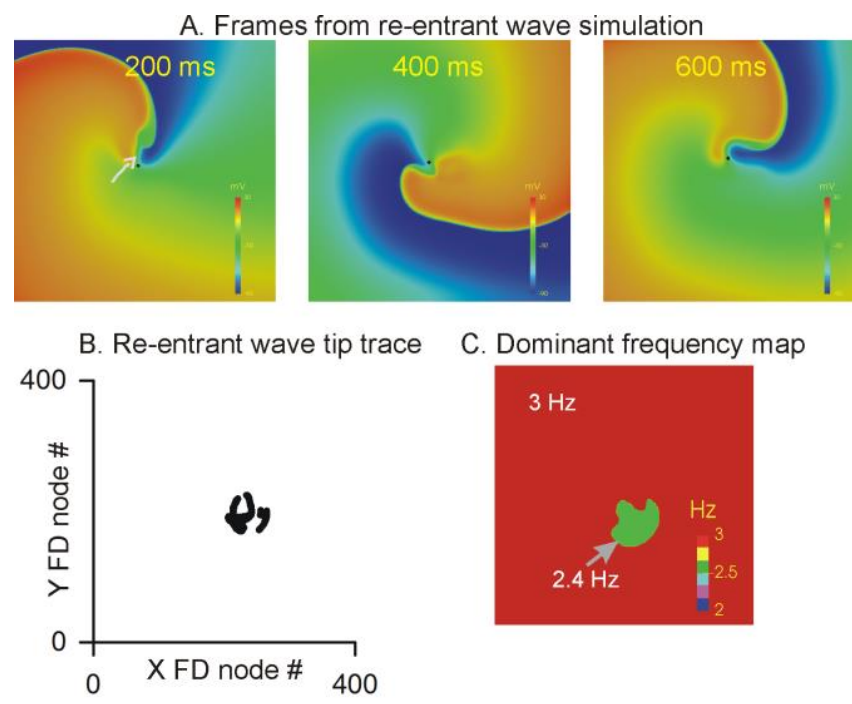

Figure 2. Pre-dialysis simulation. A: Representative frames from the 2 second simulation of a single re-entrant wave. The small dots show location of the estimated reentrant wave tip. The color bar represents a membrane potential range of $-90 \mathrm{mV}$ to $+20 \mathrm{mV}$. B: Re-entrant wave tip trace throughout the span of the simulation. C: Dominant frequency map of the 2 seconds of electrical activity.

$0.5 \mathrm{~m} / \mathrm{s}$. Within the ischemic zone, the diffusion parameter was reduced 10-fold representing a drastic reduction of ischemia induced cell-cell electrical coupling.

\section{Initial conditions and protocols to permit quantification of arrhythmia propensity.}

The 2D geometry consisted of either a homogenous normal zone sheet (pre-dialysis conditions), or a sheet with randomly distributed non-overlapping known number of ischemic zones (post-dialysis conditions). The post-dialysis geometries had 1, 10 , or 20 ischemic zones. To facilitate assessment of arrhythmogenesis, an initial single re-entrant wave (single phase singularity) using the phase distribution method as described previously [8] was used to initiate a dynamical single re-entry. In brief, a phase, $\vartheta$, between 0 and $2 \pi$ was assigned to each location in the 2D sheet using the equation:

$\theta=\tan ^{-1}\left(\frac{y-y_{0}}{x-x_{0}}\right)-\frac{2 \pi}{L}\left(\left(x-x_{0}\right)^{2}+\left(y-y_{0}\right)^{2}\right)$

where $L$ is a distance measure in the re-entrant wave kept large for a single armed re-entry, and $\left(\mathrm{x}_{0}, \mathrm{y}_{0}\right)$ is the user defined center (phase singularity) of the re-entrant wave's phase. In this study, $L=1000 \mathrm{~mm}$ and $\left(\mathrm{x}_{0}, \mathrm{y}_{0}\right)=(40 \mathrm{~mm}, 40$ $\mathrm{mm}$ ). The action potentials (Figure 1A) were assigned a phase of $\Theta=0$ which corresponded to the values of all 39 dynamical variables at time $\mathrm{t}=0$, and $\Theta=360^{\circ}$ corresponded to time $\mathrm{t}=$ $600 \mathrm{~ms}$. Using the spatial distribution obtained from equation 1 , the dynamical variables were distributed over the complete 2D sheet to initiate a single re-entrant wave. These initial conditions represent an artificially induced single tachycardia to assist with the study of genesis of multiple re-entrant waves. Whereas the physiological pacing rate of the human ventricle is around $1 \mathrm{~Hz}$, re-entrant waves often pace the heart at a much higher pacing rate. Therefore, physiological pacing was considered to be overdrive suppressed by the induced propagations. The wave dynamics were permitted to evolve for a simulated time period of 2 seconds, with the membrane potential distribution in the $2 \mathrm{D}$ model being recorded at every $1 \mathrm{~ms}$ intervals.

\section{Pre and post-dialysis simulations}

Simulations were performed under approximate pre dialysis and post-dialysis conditions. In the pre-dialysis simulation, the 2D sheet was taken to be homogenous without any ischemic zones. In the post-dialysis simulations, four cases were considered where 1,10 or 20 ischemic zones were included into the 2D sheet. Within the confines of this study, the inclusion of an increasing number density of ischemic zones are interpreted as vascular disease progression.

\section{E. Quantification of arrhythmia}

To quantify arrhythmia, two measures were computed. Firstly, the re-entrant wave tip trajectories were computed using our phase singularity detection algorithm [8, 9]. Secondly, the dominant frequency at each location in the 2D model was computed. Dominant frequency was computed using algorithms available in the FFTW3 parallel library permitting rapid as well as high throughput computation of dominant frequency maps.

\section{F. Algorithms and numerical methods}

In this study, a mono-domain formulation with no-flux boundary conditions was adopted:

$$
\begin{aligned}
& C_{m} \frac{\partial V(x, y)}{\partial t}=-I_{i o n}(x, y)+\nabla D(x, y) \nabla V(x, y) \text { in } \Omega \\
& \vec{n} \bullet \nabla V=0 \text { in } \partial \Omega
\end{aligned}
$$

where $C_{m}$ is the cardiomyocyte capacitance, $I_{\text {ion }}$ is the total current produced by the cell (both defined in [6]), $V$ is the membrane potential, $D$ is intracellular coupling defined above, $n$ is the normal at edges, and $\Omega$ is the computational domain. Wave propagation with the above equation was achieved using an operator splitting method. The cell model equations were solved numerically using an implicit method for solving ordinary differential equations. The partial differential equation part was solved using second order implicit finite difference method, encoded into a message passing interface (MPI). The maximum time step was set to $0.1 \mathrm{~ms}$. Our parallel platform is implemented in $\mathrm{C}$ language. The code was profiled using Allinea MAP software available in the Compute Canada HPC supercomputers.

\section{RESULTS}

\section{A. Homogenous pre-dialysis ventricle does not promote arrhythmia or multiple re-entrant waves}

In the pre-dialysis case (Figure 2), the re-entrant wave meander was found to be limited along its natural meander path (Figure 2, B). Whereas such a tachycardia may selfterminate in a healthy ventricle, the persistence of this re-entry 


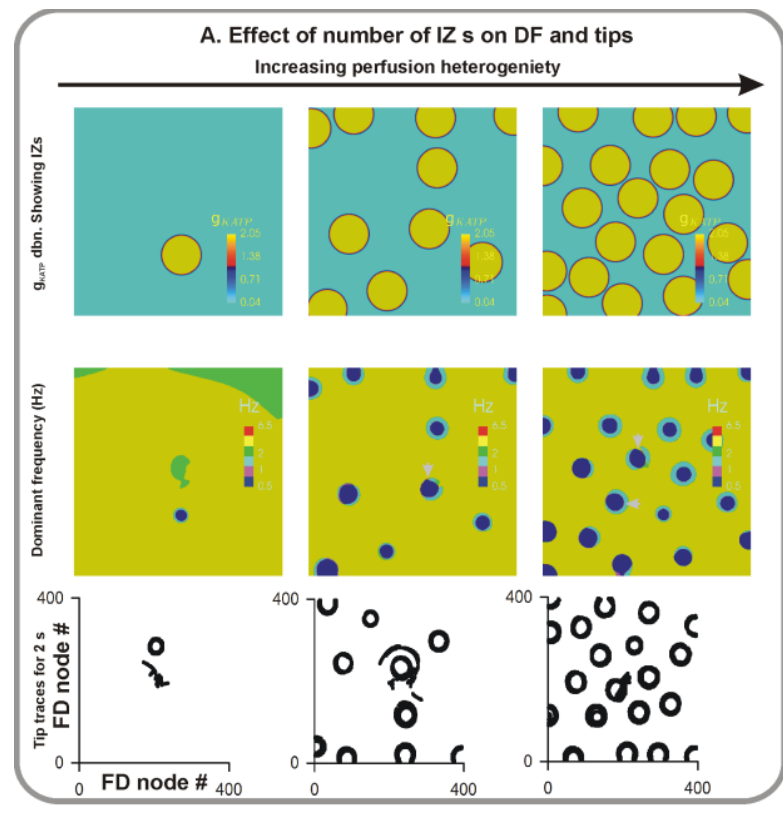

B. Evolution of tips over time

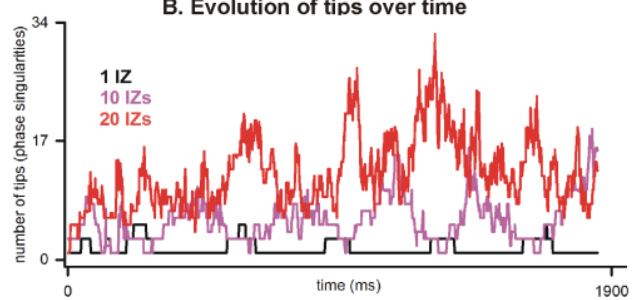

Figure 3. Post-dialysis simulation. A: Top panels show the distribution of the ischemic zones (IZs). The gradient of the I KATP conductance, $\mathrm{g}_{\mathrm{KATP}}$, is shown in the individual panels. Middle panels show the corresponding dominant frequency (DF) maps. The colour bar represents frequencies between 2 $\mathrm{Hz}$ and $7 \mathrm{~Hz}$. Bottom panels show the re-entrant wave tip patterns. B: Number of re-entrant wave tips as a function of time in the 3 cases shown where black line represents case with 1 ischemic zone, purple line represents case with 10 ischemic zones, and red line represents case with 20 ischemic zones.

was due to the slow conduction. Further, the computed dominant frequency (Figure 2, C) shows that most of the 2D sheet was paced at the period of the rotating wave of around 3 Hz. A small region was paced at a lower pacing rate of around $2.5 \mathrm{~Hz}$. This small region is enclosed by the meander pattern where the arm of the single re-entry did not excite the tissue.

\section{B. Ischemic zones' heterogeneity is a cause of arrhythmia by promoting re-entrant wave breakup.}

The results of post-dialysis simulations are illustrated in Figure 3. Increasing conduction heterogeneity was simulated by including 1, 10, or 20 ischemic zones (Figure 3, A, top panels). The dominant frequency maps (Figure 3, A, middle panels) show that when the number of ischemic zones was small (i.e. 1), the maximum pacing experienced by the tissue was around $2 \mathrm{~Hz}$. However, in the case when a significant portion of the tissue included ischemic zones, i.e. 20 zones, the maximum frequency was significantly higher at $6.5 \mathrm{~Hz}$. The fast rate of pacing occurred exclusively in the ischemic

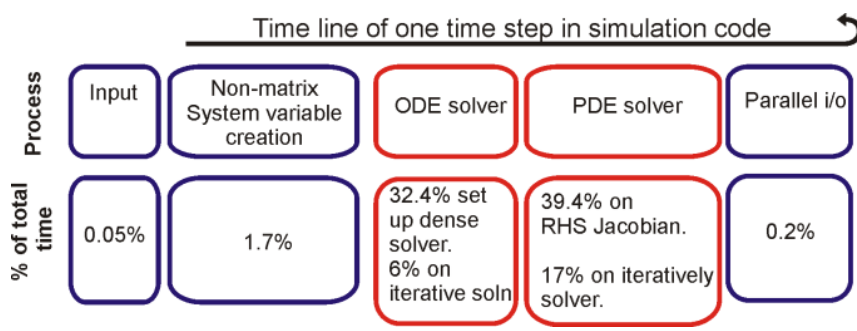

Figure 4. Efficacy profile of the code. Top panels show the main components of the MPI based code developed in our group. Bottom panels show the corresponding time taken by each component.

border zones (Figure 3, A, middle panels). Further, the tip traces of the re-entrant waves show that in addition to the induced single tachycardia, the ischemic border zone always promoted short lived but many re-entrant waves. The number of re-entrant wave tips are summarized in Figure 3, A, bottom panels and shown explicitly in Figure 3, B. The number of reentry tips oscillated between a maximum and minimum in all cases. This indicates that the induced single tachycardia was the dominant rotor driving the generation of smaller phase singularities in the ischemic border zones. However, when the number of ischemic zones was large, the induced tachycardia rapidly dissipated giving rise to erratic wave propagations. Although the number of tips was large in the 20 ischemic zones case, the time evolution still shows an oscillation which is due to the initial conditions affecting the short $2 \mathrm{~s}$ simulation. The maximum number of re-entrant wave tips induced by one ischemic zone was around 20. However, the maximum number of re-entrant wave tips induced by 10 ischemic zone patches was 20 , while in case of 20 ischemic zones it was 34 .

\section{Model code profile.}

The model code took approximately 11 hours using 24 processors to simulate one case and required $0.36 \mathrm{~GB}$ memory. To identify the computational bottlenecks, the most important functions of the code were profiled using the MAP profiler (Figure 4). At each time step iteration, it was found that the maximum CPU time was spent during the solutions of the ordinary and partial differential equations parts of the model. In both solvers, a numerical approximation of the relevant sub-systems Jacobian is computed automatically using the respective library functions. This process consumed $70 \%$ of the total time effort. The computation of the Jacobian provides the mechanism for accurate Newton iteration-based solutions to defined tolerance values. This results in the actual calculation of solution at the next time step to be $23 \%$ of total CPU effort. Unlike in previous codes, the I/O overheads in our platform were negligible.

\section{FUTURE DIRECTIONS}

\section{A. Modelling limitations}

The cell model was used to produce normal and ischemic zone action potentials. However, it is known that the human heart is transmurally heterogenous. At the spatial level, the 
model geometry used in this study represents the ventricle as a 2D sheet. However, the 3D heart has an uneven geometry and includes several heterogeneity inducing features such as fiber orientation, Purkinje stimulation network, papillary muscles, and vasculature. Ischemia is a multi-factorial condition that requires inclusion of several cellular and subcellular functional alterations for an accurate health status assessment. In future studies, the sensibility and suitability of the models, as done in case of heart cell models, will be carried out prior to their use in mechanistic simulations [10]. Ischemia is also known to affect the cardiac structure and microarchitecture, which are topics of future investigation in light of our clinical observations.

\section{B. Computational aspects}

A spectrum of computing developments are planned to permit real time patient specific assessments. In this study, the rectangular geometries that were used lend themselves to a straightforward box partitioning. It is known that realistic geometries occupy less than $30 \%$ of the total volume [11], which often leads to uneven load on processors in distributed memory machines. In addition, a larger amount of memory is required for full 3D simulations. To optimize memory use, a method for mapping of the dynamical system variables onto a 1D array was developed previously [11]. To further improve both the load balancing as well as memory use, graph theorybased partitioning will be implemented in the future. With significance to box geometry-based studies such as the present, it is relevant to provide user defined functions rather than relying on their numerical computation to improve efficiency of the codes.

\section{CONCLUSIONS AND DisCUSSION}

The main findings of this study were: 1) The electrophysiological heterogeneity in the ischemic border zones causes generation of re-entrant waves; 2) the number of ischemic zones is directly proportional to both measures of arrhythmia potential, i.e. number of re-entrant wave tips and dominant frequency.

This study presents a relationship between ischemic zones and degeneration of a single tachycardia into ventricular fibrillation. In pre-dialysis patients' hearts, the myocardium may be diseased but may also be relatively homogeneous because the serum has the opportunity to diffuse everywhere. On the other hand, post-dialysis serum content is likely to be significantly altered due to the 4 hour long dialysis induced ischemic stress. The post-dialysis refreshed serum is unlikely to permeate the complete myocardium due to a combination of vascular disease and other unidentified loss of function. Our study shows that significant fibrillation occurs when the number of ischemic zone is large (Figure 3). This finding is in line with other theoretical studies that highlighted the role of tissue spanning fibrosis [3], which is another cause for conduction heterogeneity.

Whereas a reasonable number of simulations were performed in this study, the number of modelling parameters affected by myocardial disease is large. It can be appreciated that real time detailed simulations may become possible when issues such as identified in Figure 4, as well as other hitherto unidentified issues are addressed. Further to the algorithmic developments, it is also necessary to exploit the upcoming GPU and hybrid technologies to accelerate simulations.

Cardiac ischemia is a hotly studied clinical condition using clinical, experimental, and computational approaches [12]. The ongoing basic science efforts in our clinicalcomputational group reflect the necessity to advance our knowledge of this widespread clinical condition that commonly occurs in our hospitals. It is evident that extension of the existing technology to benefit the clinical sciences requires both, intense modelling and specific experimental and imaging input to personalize the outcomes. Mechanistic computational modelling is expected to play a role within health sciences research and practice, which calls for extensive cross-disciplinary collaboration.

\section{ACKNOWLEDGMENT}

We thank Compute Canada for HPC resources and Canarie (RS3-111) for support. We thank LHSC IT department for their support. We thank members of LHSC for critical discussions.

\section{REFERENCES}

[1] J. O. Burton, S. Korsheed, B. J. Grundy, and C. W. McIntyre, "Hemodialysis-induced left ventricular dysfunction is associated with an increase in ventricular arrhythmias," Ren Fail, vol. 30, pp. 701-9, 2008.

[2] C. W. McIntyre, J. O. Burton, N. M. Selby, L. Leccisotti, S. Korsheed, C. S. Baker, and P. G. Camici, "Hemodialysis-induced cardiac dysfunction is associated with an acute reduction in global and segmental myocardial blood flow," Clin J Am Soc Nephrol, vol. 3, pp. 19-26, Jan 2008.

[3] E. Vigmond, A. Pashaei, S. Amraoui, H. Cochet, and M. Hassaguerre, "Percolation as a mechanism to explain atrial fractionated electrograms and reentry in a fibrosis model based on imaging data," Heart Rhythm, vol. 13, pp. 1536-43, Jul 2016.

[4] S. Dutta, A. Minchole, T. A. Quinn, and B. Rodriguez, "Electrophysiological properties of computational human ventricular cell action potential models under acute ischemic conditions," Prog Biophys Mol Biol, Feb 202017.

[5] J. Carro, J. F. Rodriguez, P. Laguna, and E. Pueyo, "A human ventricular cell model for investigation of cardiac arrhythmias under hyperkalaemic conditions," Philos Transact A Math Phys Eng Sci, vol. 369, pp. 4205-32, Nov 132011.

[6] J. M. Ferrero, J. Saiz, J. M. Ferrero, and N. V. Thakor, "Simulation of action potentials from metabolically impaired cardiac myocytes. Role of ATP-sensitive K+ current," Circ Res, vol. 79, pp. 208-21, Aug 1996.

[7] S. R. Kharche, A. So, F. Salerno, T. Y. Lee, C. Ellis, D. Goldman, and C. W. McIntyre, "Computational Assessment of Blood Flow Heterogeneity in Peritoneal Dialysis Patients' Cardiac Ventricles," Front Physiol, vol. 9, p. $511,2018$.

[8] S. R. Kharche, I. V. Biktasheva, G. Seemann, H. G. Zhang, and V. N. Biktashev, "A Computer Simulation Study of Anatomy Induced Drift of Spiral Waves in the Human Atrium," Biomed Res Int. 2015; 2015: 731386. Epub 2015/10/26, 2015.

[9] S. R. Kharche, I. V. Biktasheva, G. Seemann, H. G. Zhang, J. C. Zhao, and V. N. Biktashev, "Computational Modelling of Low Voltage Resonant Drift of Scroll Waves in the Realistic Human Atria," Functional Imaging and Modeling of the Heart (Fimh 2015), vol. 9126, pp. 421-429, 2015.

[10] E. T. Chang, M. Strong, and R. H. Clayton, "Bayesian Sensitivity Analysis of a Cardiac Cell Model Using a Gaussian Process Emulator," PLoS ONE, vol. 10, p. e0130252, 2015.

[11] S. Kharche, G. Seemann, L. Margetts, J. Leng, A. V. Holden, and H. Zhang, "Simulation of clinical electrophysiology in 3D human atria: a highperformance computing and high-performance visualization application," Conc. Comp. Exp., vol. 20, p. 10, 2008.

[12] B. Rodriguez, N. Trayanova, and D. Noble, "Modeling cardiac ischemia," Ann N Y Acad Sci, vol. 1080, pp. 395-414, Oct 2006. 